\title{
O Estado da Arte em Pesquisas sobre Humanidades Digitais no Brasil
}

\section{Luis Felipe Rosa de Oliveira ${ }^{1}$ e Dalton Lopes Martins ${ }^{2}$}

1 Bolsista de Pesquisa pela Universidade Federal de Goiás, Brasil. E-mail: luisfelipeprf@gmail.com

2 Doutor em Ciências da Informação pela ECA-USP, Mestrado em Engenharia da Computação pela Universidade Estadual de Campinas, graduação em Engenharia Elétrica pela Universidade Estadual de Campinas. Professor no curso de Gestão da Informação e no Programa de Pós-graduação em Comunicação PPGCOM (Mestrado) da Faculdade de Informação e Comunicação da Universidade Federal de Goiás, Brasil. E-mail: dmartins@gmail.com

RESUMO: O conceito de humanidades digitais vem sendo utilizado recentemente para descrever como o contexto digital/tecnológico tem modificado a forma como as pesquisas, estudos e projetos na área são trabalhados. Mas além disso, em uma visão mais abstrata, este tema também representa a ânsia por entender como a sociedade se comporta diante da recente e crescente disponibilidade tecnológica que está presente a sua volta. Assim, este artigo se preocupa em entender como a produção cientifica em relação a este tema está disposta no contexto brasileiro, e entrega produtos analíticos que comprovam a baixa produção, revelando a necessidade de atenção ao tema.

Palavras-chave: Humanidades Digitais. Pesquisa. Produções. Sociedade.

\section{The State of Art in Researches about Digital Humanities in Brazil}

\begin{abstract}
The concept of digital humanities has been recently used to describe how the digital/technological context has changed the way of how the researches, studies and projects in the area are worked. But beyond that, in a broader vision, this theme also represents the craving to understand how the society behaves in front of the recent and growing technological availability that is preset around it. So, this article worries in understand how the scientific production related at this theme is disposed in the Brazilian context, and bring analytical products that proves the low production, revealing the necessity of attention about that theme.
\end{abstract}

Keywords: Digital Humanities. Research. Productions. Society.

\section{INTRODUÇÃO}

Se pensarmos que a sociedade da forma como ela se apresenta hoje, se deu a partir das diversas variações dos contextos históricos vividos pela humanidade, é comum entender que, o design das coisas à sua volta, os processos e tarefas executadas nas mais diversas rotinas, tudo que o ser humano viveu em seu desenvolvimento foi aperfeiçoado e estruturado de acordo com os complexos contextuais vividos pelas 
pessoas.

Dessa forma, pensando nos períodos da estruturação social humana, historicamente, houveram revoluções tanto econômicas, quanto políticas, sociais, e recentemente tecnológicas. Todas a partir de certo ponto de vista indicando uma mudança pontual no modo de como as pessoas se comportam em sociedade.

A mudança de comportamento social, significa que o modo de vivência atual já não é mais o mesmo de antes. Pensar desta forma, revela uma dinamicidade que nos proporciona o entendimento de que vários fatores condicionaram tais mudanças, e talvez por sua abstração e complexidade, seja impossível reunir todos as causas de certa mudança.

Porém, como é proposto neste artigo, e é um tema de ampla discussão e busca por entendimento atualmente, o foco a ser tratado dentre estes fatores diretamente ligados ao comportamento social é o meio digital, composto por seus diversos tipos de tecnologias e metodologias de apropriação.

O contexto digital que têm seu início por volta dos anos 1970, teve, em matéria de evolução, dentro de mais ou menos quarenta anos, um crescimento exacerbado, com evoluções tecnológicas e mudanças consideráveis no comportamento do mercado econômico, além disso, a sociedade se apresenta conectada de forma global.

Inúmeros núcleos sociais foram afetados com a inserção deste ambiente que proporciona novas maneiras de como fazer, e novos fazeres. Um destes núcleos, que é o ambiente que compõe o objetivo deste trabalho, é o meio da pesquisa, mais especificamente pesquisa em humanidades.

O contexto das humanidades pode ser compreendido por diversas áreas de conhecimento, dentre elas: história, literatura, filosofia, artes, educação, ciências sociais, música e etc. Áreas que são estruturadas de certa forma em pesquisas abstratas, de cunho mais qualitativo, partindo da análise mais subjetiva do pesquisador.

O motivo de este trabalho se direcionar para o escopo das humanidades em específico, se dá na necessidade de entender como o cenário digital é tratado e influencia este arranjo mais abstrato e subjetivo da pesquisa acadêmica.

O estudo das influências, possibilidades e composição do digital nas humanidades dá nome a uma área específica chamada humanidades digitais (HD), que a priori é uma área que, de forma geral, busca entender o escopo acima definido para este trabalho: como é a relação entre as variáveis do meio digital e as metodologias de desenvolvimento das áreas que compõe as humanidades.

Antes de aprofundar na conceituação de humanidades digitais, é interessante entender os conceitos "digital" e "humanidades" de forma separada, mais ainda inseridos neste contexto de complementaridade entre eles.

Segundo Gardner e Musto (2015), os estudos em humanidades, procuram identificar como a linguagem se desenvolve de forma temporal, sua origem, seu processo de estruturação, e o que pode vir a ser, além disso, um dos principais objetivos destes estudos é entender o mundo que as pessoas têm concebido para si. 
Sendo assim, é possível interpretar que a composição das humanidades se dá a partir da necessidade de entender a presença e ações do ser humano em seu meio de existência, como já contextualizado anteriormente.

Já sobre o digital, ainda segundo Gardner e Musto (2015), ele tem a capacidade de potencializar o montante de informações que as pessoas têm acesso, possibilitandoas também, através das máquinas de busca e outras tecnologias, a capacidade de processá-las, produzir novas informações, e conectá-las de maneira mais acessível às pessoas em geral.

Ou seja, o digital pode ser entendido como a base ou meio ferramental e relativamente metodológico que auxilia e intensifica as capacidades humanas de relacionamento, desenvolvimento, e apropriação das representações de sua realidade.

Unindo os dois conceitos novamente e voltando para o termo principal: humanidades digitais, vários pesquisadores, em suas publicações, expõe sua visão conceitual do termo, todas em torno do núcleo principal que tange à relação entre o digital/tecnológico e as humanidades, em seu cunho social e como área de pesquisa abstrata.

Com uma visão mais sistêmica do conceito de humanidades digitais, Alan Liu (2016) apresenta a HD como uma área geral que engloba duas outras sub-áreas de estudo: os estudos sobre as novas mídias e os estudos sobre tecnologias de informação. Assim, sua visão representa as humanidades digitais como um composto de outras áreas de estudo relativas ao meio digital e humano, que ao mesmo tempo que às engloba, às incrementa com um pensamento conjugado entre elas.

Partindo de uma visão mais sucinta e direta, o dicionário de termos literários e teoria literária de Cuddon (2013) explicita a seguinte definição para humanidades digitais:

\footnotetext{
"Um amplo campo multidisciplinar dedicado a entender a intersecção entre a tecnologia da informação e as humanidades tradicionais. As áreas cobertas incluem a digitalização de textos e o uso de computadores como ferramentas para linguística e análise de texto, apesar de que seu escopo em potencial é considerado mais vasto entre seus praticantes. " (CUDDON, 2013, p.204)
}

Ou seja, novamente surge a inferência do elo entre as áreas tecnológica e humana dentro de um contexto mais específico como a pesquisa, ou trabalhos afins destas duas áreas.

Para Hayles (2012), existe uma certa interposição entre o ambiente tecnológico e o contexto das humanidades, sendo que existe também, uma preocupação sobre o estudo tradicional das humanidades e sua ideologia "limitada", de modo que as Humanidades Digitais têm de ser vistas como mais do que tecnologias ou ferramentas e sim uma mudança de paradigma existente na área de humanidades tanto na tradicional quanto na digital daqui para frente. 
Kramer (2012), têm uma descrição de humanidades digitais parecida com a de Hayles, porém mais focada no profissional, ele argumenta que os profissionais que atuam no contexto das humanidades digitais se encontram em um paradigma entre os estudos abstratos de suas variadas temáticas e a sua sistematização em sistemas computacionais. Sendo que este profissional não deve ser visto somente pela análise humanística auxiliada quantitativamente pela tecnologia, mas como um profissional que adiciona a essência humana à interpretação e observação de forma qualitativa aos dados e informações de sua análise.

Por último, David Berry, levanta uma abordagem mais aproximada do conceito de humanidades digitais a ser proposto neste artigo revelando uma perspectiva do conceito um pouco mais distante da academia, ele explicita sobre a realidade mediada através dos meios digitais, e como a sociedade em geral, em sua cultura diversificada, tem apresentado mudanças quanto ao contexto tecnológico que nos é propiciado atualmente.

Sendo assim, Berry (2011), traz a visão de humanidades digitais como um modo de se pensar as mudanças que ocorrem tanto no meio da pesquisa quanto nos movimentos sociais em geral que são condicionadas pela base tecnológica presente atualmente.

Desta forma, a partir de todas estas conceituações, é possível identificar o potencial contexto das humanidades digitais, que é representada pelo campo de estudo e pesquisa, com estruturação tecnológica e objeto humano, que contém paradigmas entre o meio tecnológico e o meio humanístico tradicional de pesquisa.

Para além desta caracterização do termo, é possível inferir também, uma inclinação para as questões sociais, e como a sociedade é afetada pela inserção deste contexto digital, já que, como já referenciado por Gardner e Musto, um dos papeis fundamentais das humanidades é entender o mundo que as pessoas criam para si.

Partindo destes preceitos, o conceito que mais se adequa a proposta deste material, é que as humanidades digitais, são um conjunto de campos de estudos humanosociais, que quando inseridos em um contexto digital/tecnológico, sofrem modificações metodológicas e estruturais, de modo que, seu objetivo principal, é identificar como as pessoas em sociedade se comportam inseridas neste contexto quanto a apropriação e construção de um mundo para si mesmas.

Utilizando então, este conceito como base, é importante elucidar a importância do estudo em humanidades digitais em países emergentes, como o Brasil. Ao desenvolver pesquisas e projetos sobre humanidades digitais, é possível aplicar políticas específicas de inserção digital na educação, incentivos à ciência e tecnologia mais diretos e concisos, além de promover no geral, um equilíbrio entre a estrutura tecnológica e social do país.

Sendo assim, um primeiro passo, mais sucinto e direto, seria responder à questão: como o conceito de humanidades digitais se situa na produção cientifica no Brasil atualmente? 
A partir da resposta para esta pergunta, será possível então, obter uma indicação de como o tema está sendo estudado no Brasil, e se já existe um bom conjunto de produções relacionadas a ele, ou se ainda é timidamente tratado pelos pesquisadores do país.

O que nos leva ao principal objetivo e produto deste artigo: mapear o estado da produção científica sobre humanidades digitais no Brasil, utilizando repositórios de publicações científicas e o diretório de grupos de pesquisa do CNPQ.

\section{METODOLOGIA}

A identificação situacional da pesquisa e desenvolvimento cientifico sobre determinado tema pode ser realizada de diferentes formas utilizando diferentes tecnologias e bases de consulta.

Para este trabalho, o ambiente a ser consultado será a Internet, e as bases de divulgação de produção científicas que nela existem, as ferramentas para análise serão software que permitem o tratamento e a produção de visualizações estratégicas para o objetivo desta pesquisa.

Para as fontes de informações sobre a produção científica do Brasil, foram definidas seis bases de consulta ${ }^{1}$ : o Google Acadêmico, interface de pesquisa do Google que retorna informações sobre produções científicas, citações e autores; o portal de periódicos da CAPES (Coordenação de Aperfeiçoamento de Pessoal de Nível Superior), que é um portal que agrega os artigos científicos publicados nos periódicos da CAPES; o Diretório de Grupos de Pesquisa do CNPQ (Conselho Nacional de Desenvolvimento Científico e Tecnológico), que é um portal que permite consultar todos os grupos de pesquisa do Brasil cadastrados no CNPQ; Scielo Brasil, que se propõe a ser uma biblioteca científica eletrônica on-line, com seu desdobramento para as produções do Brasil; o BDTD (Biblioteca Digital Brasileira de Teses e Dissertações), que reúne um montante de teses e dissertações realizadas no país; e a Scopus que é uma base de dados bibliográficos para jornais e revistas acadêmicos.

A partir deste levantamento e definição de bases de consulta, pretende-se realizar um mapeamento das produções cientificas do Brasil, sejam elas artigos, produções acadêmicas em geral, e mesmo grupos de pesquisa envolvidos com o tema.

Todas estas bases permitem a inserção de palavras-chave para consulta. Quando a consulta por determinada palavra é realizada, ela pode ser procurada tanto no corpo do documento, quanto no título, ou em suas descrições, resultando assim, em documentos que contenham tal palavra em seu contexto.

Sendo assim, o termo chave escolhido para a pesquisa nas bases foi "Humanidades Digitais", que por estar escrito em português já filtra resultados de países com línguas diferentes.

\footnotetext{
${ }^{1}$ A consulta sobre o tema Humanidades Digitais nestas bases foi realizada no dia 18 de julho de 2016 , sem que os resultados se referem a todos os documentos produzidos até esta data.
} 
O método de busca nas bases foi diferente de acordo com cada uma: no diretório de grupos de pesquisa, foi pesquisado Humanidades Digitais, sem aspas ${ }^{2}$ com todos os filtros por padrão, selecionando somente os grupos certificados e atualizados; no portal Scielo Brasil, foi pesquisado o termo Humanidades Digitais tanto com e sem aspas em todos os campos do documento; no BDTD, foi pesquisado o termo com e sem aspas no título de no assunto do documento; no portal de periódicos da CAPES, foi pesquisado Humanidades Digitais com aspas, refinando a busca pelo título; na base Scopus, foi pesquisado Humanidades Digitais com aspas, com todos os filtros por padrão.

Para retirar as informações da base do Google Acadêmico, foi utilizado um software denominado Publish or Perish, ele permite que a base seja consultada por ele, e organiza os resultados em formato tabular, facilitando a visualização e análise. Nele o termo Humanidades Digitais foi pesquisado com aspas e reduzindo o campo de busca para os títulos dos documentos.

Obtidos todos os resultados das análises, eles foram sistematizados em formato de planilha, e utilizando o software Libre Office Calc, foi possível sistematizar, tratar e gerar dados analíticos dos resultados de busca.

Para desenvolver as visualizações, foram utilizados dois softwares diferentes: para os gráficos mais comuns como barras e rosca, foi utilizado o Microsoft Office Excel, e para análise espacial dos grupos de pesquisa existentes no Brasil, foi utilizado o Tableau Public, que permite a visualização em mapa.

\section{ANÁLISE E RESULTADOS}

Os resultados obtidos da pesquisa realizada nas fontes de informação citadas acima apresentaram pouca relevância em produções científica no Brasil, a seguir eles podem ser visualizados graficamente (Figura 1):

Figura 1 - Número de Resultados por Base Pesquisada. Fonte: Elaborado pelo Autor.

\section{Número de Resultados por Base Pesquisada}

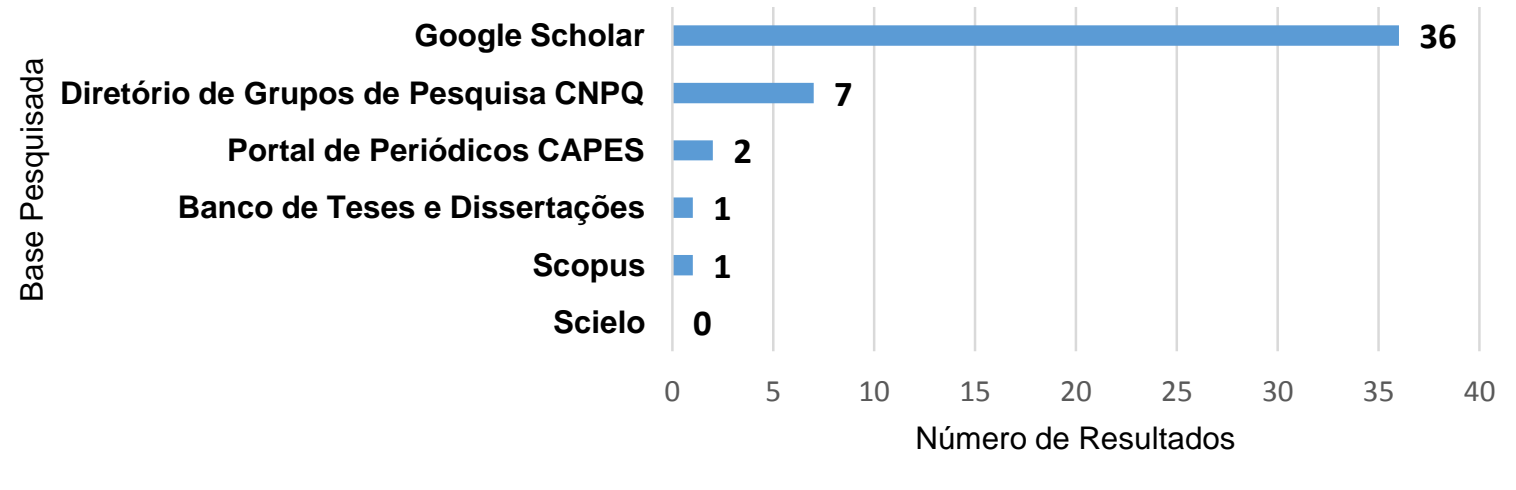

\footnotetext{
${ }^{2}$ Quando um termo é pesquisado com aspas, por exemplo "Humanidades Digitais", o mecanismo de pesquisa procura pelo termo composto como ele é, sem divisão entre as duas palavras.
} 
Como, visualizado, a base do Google Acadêmico retornou mais resultados para a busca em Humanidades Digitais, sendo que, os documentos retornados, são compostos de livros, artigos e até páginas de publicações na internet; a pesquisa no diretório dos grupos de pesquisa retornou sete grupos de pesquisa existentes pelo Brasil com temáticas relacionadas a humanidades digitais; já o portal de periódicos da CAPES, o BDTD, a Scopus e a Scielo, retornaram resultados abaixo de três documentos científicos.

Essas fontes de informação que obtiveram resultados menores que três documentos (Scielo, Portal de periódicos da CAPES e Scopus), permitem o pensamento potencial de que, elas indicam que, os periódicos indexados por estas bases têm muito pouca referência sobre o tema de humanidades digitais, denotando também, o baixo grau produções relacionadas ao tema.

Quanto aos resultados sobre o diretório de grupos de pesquisa do CNPQ, o número de sete grupos no Brasil relacionados ao tema indica uma quantidade relativamente boa de conjuntos de pessoas que estudam se não o tema específico, temas que matem relação com ele no Brasil.

Sobre os resultados obtidos da base do Google Acadêmico através do Publish or Perish, eles resultaram em 36 documentos com referência ao tema, estes documentos, como mencionado anteriormente, são compostos desde artigos científicos a publicações em páginas na internet.

Outra observação importante a ser feita sobre a base do Google Acadêmico, é que, como o termo de consulta foi utilizado em português, foram recuperados pela base, tanto publicações do Brasil como de Portugal.

Sendo assim, o resultado da base de 36 publicações relacionadas ao tema, não é totalmente referente às publicações do Brasil, assim, como a figura 2 apresenta, no contexto nacional brasileiro, foram obtidas 21 produções relacionadas ao tema.

Figura 2 - Quantidade de Resultados de Consulta no Google Acadêmico por País. Fonte: Elaborado pelo Autor

\section{Quantidade de Documentos por País}

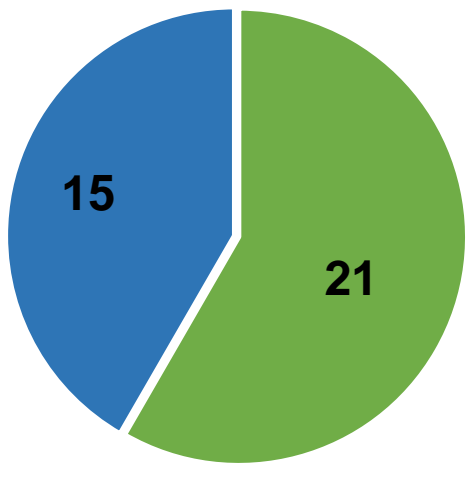

- Brasil

- Portugal

Assim, ao contemplar os resultados sobre os materiais relacionados ao temas nas diferentes fontes de informações selecionadas, é possível chegar a duas conclusões: a 
primeira de que esse baixo indicador de publicações denota uma importante necessidade de atenção para o campo: de que o tema ainda é pouco trabalhado em âmbito brasileiro, isso confirma o que já era esperado pela recente atenção ao tema, porém, como já mencionado, em um país preocupado com seu desenvolvimento, a pouca visibilidade ao tema pode ser considerada preocupante.

O segundo ponto, que define o restante das análises deste artigo, é que como as bases Scielo, Scopus, BDTD e o portal da CAPES retornaram uma quantidade de resultados muito pequena para inferências, utilizamos somente o Google Acadêmico com vinte e uma produções Brasileiras e a base dos grupos de pesquisa do CNPQ, composta de sete grupos de pesquisa Brasileiros.

As análises da base do Google Acadêmico se dividiram em 2 momentos, o primeiro na tentativa de verificar a taxa de publicações sobre o tema por ano no Brasil (Figura 3), e o segundo apresentando de forma geral, quais os temas que são contemplados pelas publicações (Figura 4).

Sobre a quantidade de publicações por ano sobre Humanidades Digitais no Brasil, a base do Google Acadêmico acompanhou um crescimento de um para 9 entre os anos de 2011 e 2013, sendo que nos anos de 2014 e 2015 e houve uma quede de cinco e quatro publicações respectivamente, já no ano de 2016 até a data da consulta, não houve nenhuma publicação referente ao tema no Brasil (Figura 3).

Figura 3 - Quantidade de Publicações por ano em Humanidades Digitais - Google Acadêmico. Fonte: Elaborado pelo Autor.

\section{Quantidade de Publicações por Ano}

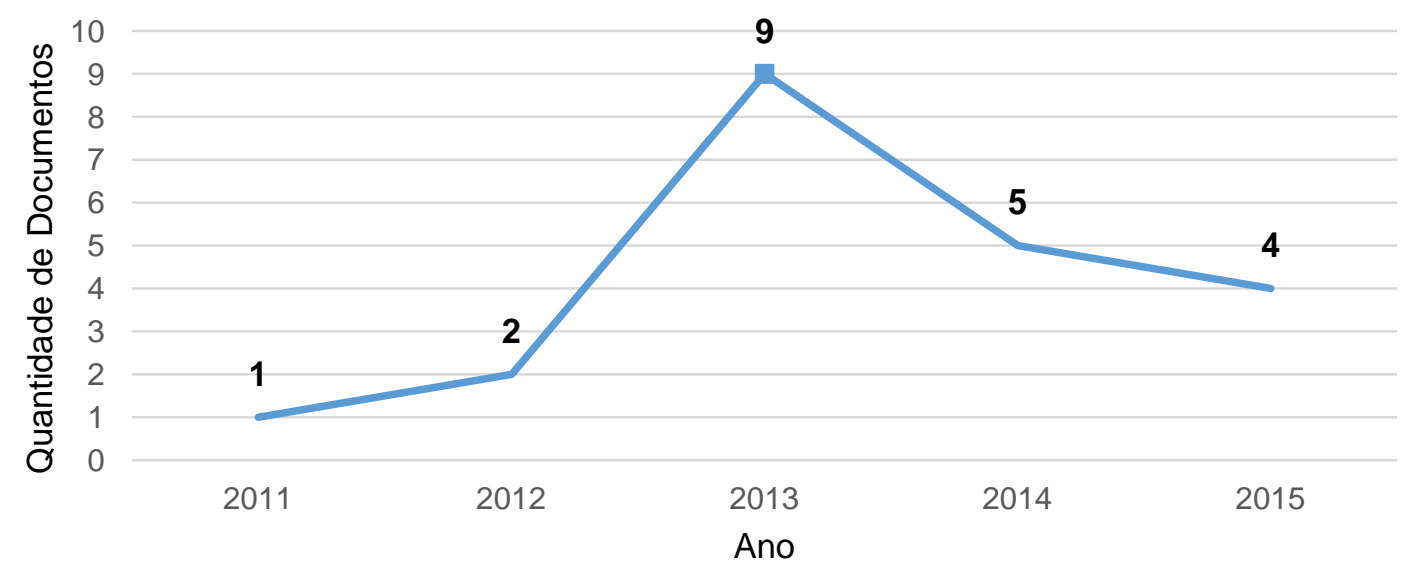

Sobre as temáticas das produções resultantes da consulta na base, foi possível, de maneira superficial, identificar alguns assuntos tratados: páginas na internet sobre a associação das humanidades digitais e seminário sobre o tema, publicações que fazem referência a áreas como ciência da informação, cultura, filologia, história e tecnologias da informação e casos sobre a contextualização do tema em bibliotecas.

Essas temáticas fazem alusão às conceituações dos vários autores citados anteriormente, o que reforça a estrutura do campo das humanidades digitais, e podem ex- 
plicar também a característica iniciante das pesquisas brasileiras quanto ao tema, que ainda está sendo trabalhado em conjunto com diversos outros temas já bem estruturados no âmbito da pesquisa brasileira.

Uma observação importante, é citar a página do blog HD.br ${ }^{3}$, que faz publicações sobre humanidades digitais na internet, que das 21 publicações sobre o tema no Brasil ela foi responsável por treze, o que indica um ambiente importante de discussão e referência ao tema no país.

Adentrando na análise dos grupos de pesquisa presentes no Brasil que trabalham com pesquisa relacionadas a humanidades digitais, foram tratados dois pontos de análise: o primeiro sobre a quais áreas cientificas eles estão conectados (Figura 4) e o segundo sobre a localização espacial de seus núcleos de pesquisa no país (Figura 5).

Com relação às áreas de pesquisa as quais os grupos estão relacionados, foram identificadas cinco áreas de estudo: Linguística (Grupo de Estudos em Linguística Computacional, Corpus e Humanidades Digitais e Grupo de Pesquisa em Linguística de Corpus), Letras (Estudos Interdisciplinares em Humanidades Digitais, Filologia e Acervos de Escritores e Literatura e mobilidades sociais e culturais), História (Hímaco - História, Mapas e Computadores), Ciência da Informação (Humanidades Digitais: ciência, tecnologia e memória) e Comunicação (Humanitas Digitalis). Todas áreas que compõe de certa forma o contexto das humanidades, e como conceituado, áreas que tiveram forte impacto com a influência das tecnologias da informação.

Figura 4 - Número de Grupos de Pesquisa por Área. Fonte: Elaborado pelo Autor.

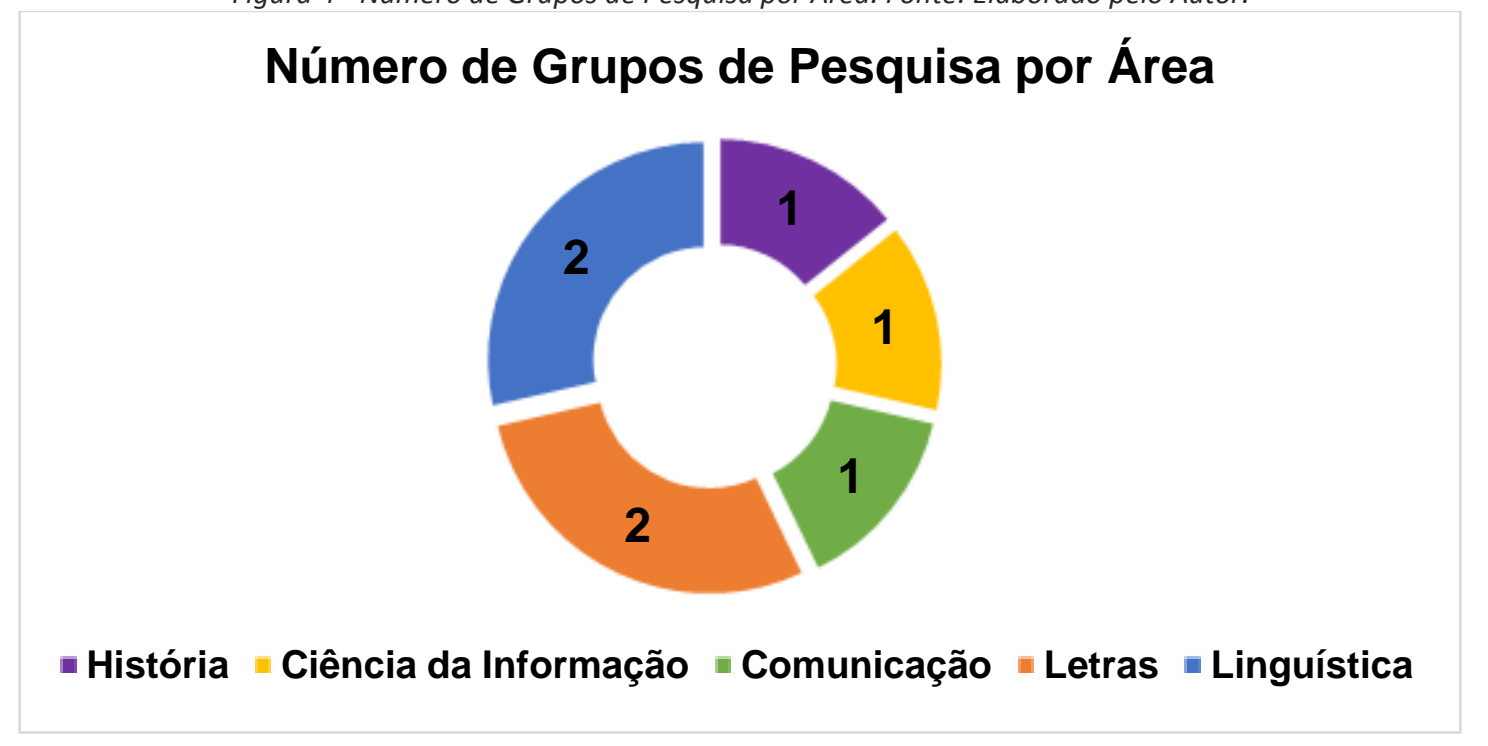

As áreas com mais grupos de estudo relacionados a elas foram Linguísticas e Letras, com dois grupos cada, Berry (2011, pgs. 3 e 4) cita sobre dois tipos de ondas que definem os momentos das humanidades digitais: a primeira onde se refere aos estudos em humanidades através de repositórios digitais, textos e etc. e a segunda relata

${ }^{3}$ http://hdbr.hypotheses.org/ 
sobre o uso de ferramentas tecnológicas para análise de matérias eletrônicos como a literatura eletrônica, a fiç̧ão interativa e etc.

Ou seja, as áreas de linguística e letras terem mais representatividade sobre as outras já é esperado no contexto deste tema, já que esse paradigma entre os processos de pesquisa tradicionais e a influência recente das capacidades digitais pode ser muito constante nestas áreas.

A presença dos grupos de estudos nas áreas de história, comunicação, e ciência da informação também podem significar como o meio de humanidades digitais vem se apresentando no Brasil, a informação de que existem grupos de pesquisa nestas áreas podem indicar que são as áreas que mais têm se identificado com as propostas do tema.

Sobre a localização geográfica dos grupos de pesquisa, como pode ser visualizado na figura 5, temos dois grupos em São Paulo, dois na Bahia, um em Pernambuco, um no Paraná e mais um no Rio de Janeiro.

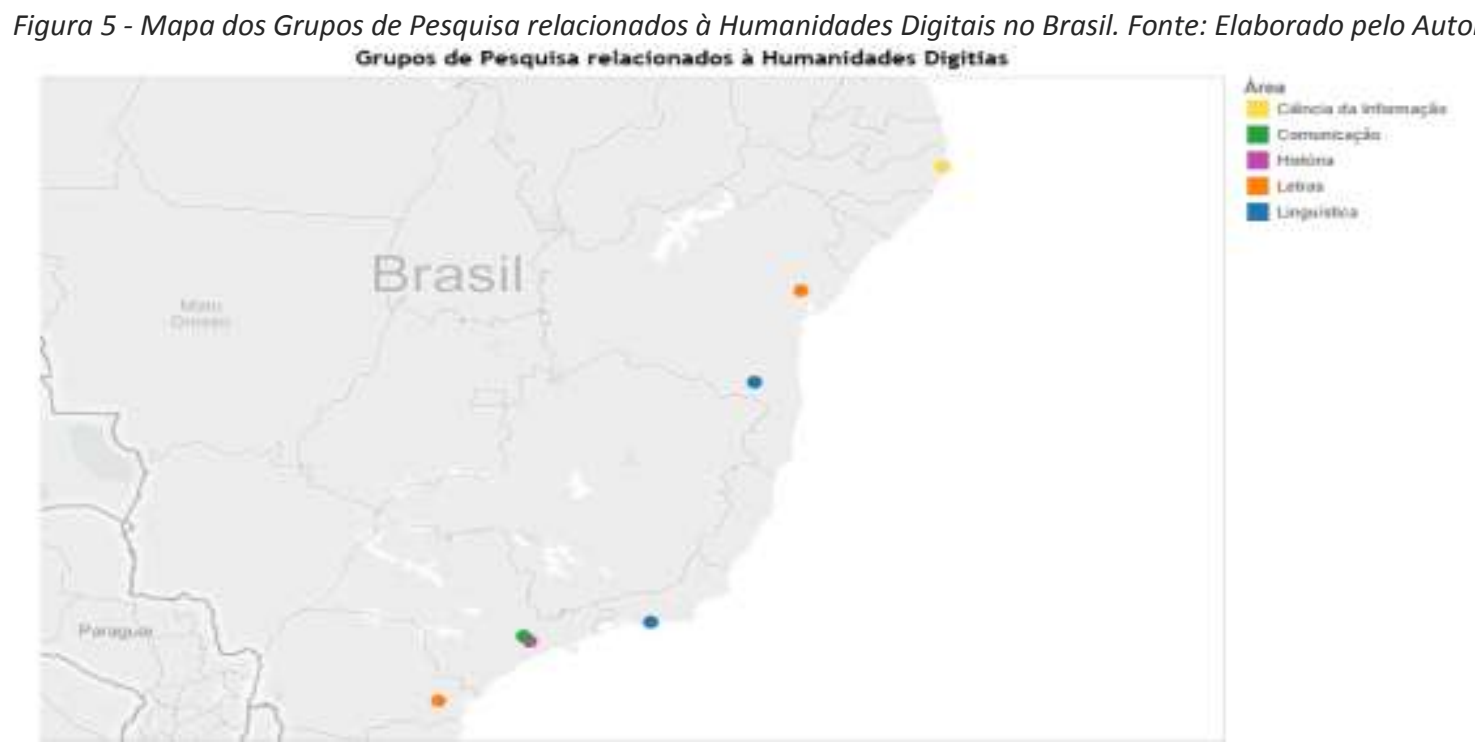

Estes pontos, podem ser visualizados como os pontos iniciais de pesquisa em humanidades digitais no Brasil, e com o tempo e avanços em pesquisas e projetos, podem se tornar os pontos de referência nacional no tema, o que indica potenciais núcleos de desenvolvimento em HD no Brasil.

\section{CONSIDERAÇÕES FINAIS}

Após a conclusão das análise e verificação dos resultados, temos três considerações importantes a serem feitas sobre a pesquisa: a primeira é que a conceituação sobre Humanidade Digitais, no que se refere a influência dos meios digitais nas áreas de estudo das humanidades se confirmou de acordo com a análise de áreas dos grupos de estudos e temáticas das publicações do Google Scholar. 
Segundo, foi confirmado que a quantidade de publicações sobre o tema é muito baixa, o que resulta em certa preocupação, já que, por mais que humanidades digitais seja um tema recente, ele dialoga com a prospecção de introdução às tecnologias e meios digitais que potencializam os estudos e o desenvolvimento tanto do meio da pesquisa quanto do meio social.

E terceiro, a apresentação dos grupos de pesquisa e a identificação do blog de humanidades digitais nos apresente alguns HUB's de produção e desenvolvimento importantes sobre o tema, sendo possível a reunião dos profissionais destes núcleos, de forma que possam trocar experiência e formar parcerias que fortaleçam a relevância do tema no país.

Sobre a pesquisa realizada de forma geral, ela demonstra de forma analítica e com base nas fontes de informação utilizada, a situação da produção científica sobre humanidades digitais no Brasil, e revela uma necessidade de dar mais relevância ao tema em âmbito nacional.

Além disso, a pesquisa também já pode ser considerada como uma aplicação metodológica em humanidades digitais, já que pelas ferramentas utilizadas, pelo contexto digital e computacional no qual foi concebido, além de considerar os resultados analíticos como relevantes para responder ao questionamento proposto, se mostra a utilização do digital/tecnológico para aturar em propósitos de pesquisa utilizados também nas humanidades.

Para complementar este trabalho, outros trabalhos de aprofundamento temático e de análises de temas complementares à temática de humanidades digitais podem ser realizados com o fim de proporcionar caminhos estratégicos para que a fundamentação do tema no país ocorra.

No mais, é nítida a necessidade de mais referências nacionais sobre o tema no Brasil, de forma que não só as áreas de estudo de humanidades se fortalecem no meio digital, mas também, que seja possível entender melhor os movimentos e comportamentos sociais que acontecem com essa influência tecnológica.

\section{REFERÊNCIAS}

Berry, D. M. The Computational Turn: Thinking About the Digital Humanities. Culture Machine, Vol. 12, pgs. 1-22. 2011.

Cuddon, J. A. A Dictionary of Literary Terms and Literary Theory, Fifht Edition, WileyBlackwell, British Library. 2013.

Gardiner, E. Musto, R. G. The Digital Humanities: A Primer for Students and Acadêmicos. Cambrydge University Press. New York. 2015.

Hayles, N.K. How We Think: Digital Media and Contemporary Technogenesis, Chicago: University of Chicago Press. 2012.

Kramer, M. J. What Does Digital Humanities Bring to the Table? Acessado em 21/07/2016. Disponível em: <http://www.michaeljkramer.net/cr/what-does-digital- 
humanities-bring-to-the-table/> 2012.

Liu, AY. The State of the Digital Humanities: A Report and Critique. Arts and Humanities in Higher Education, vol.11, pgs. 1-49. 2012.

Artigo recebido em 27 de julho de 2016.

Aprovado em 01 de agosto de 2017. 